\title{
SEALING ROHACELL TO PREVENT FLAKING
}

Do Engineering Note: $3823.113-E N-499$

August 12, 1998

Stephanie McDade

Approved By

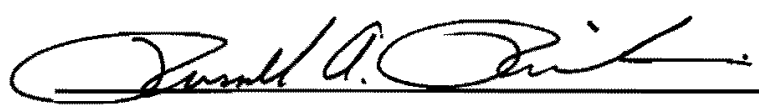




\begin{abstract}
The light, rigid foam, Rohacell, is an insulator in the cassettes for the VLPC cryostat. Focusing attention on the flaking of the Rohacell, the fear was that the Rohacell particles would interfere with the electrical equipment of the cassette. Several methods were tested to find a way to prevent the flaking, including cleaning, coating, and heating the Rohacell. Various coatings were used in trying to seal the Rohacell: Krylon, red insulating epoxy, vacuum sealant and Dapco 2030. After immersing the samples in liquid Nitrogen and letting them warm to room temperature ten times, the results were clear. Using nothing produced 8 larger flakes and countless smaller flakes. The sample coated with the Krylon exhibited 5 larger flakes and 25 smaller particles. The vacuum sealant coated specimen only generated 5 smaller flakes. When tapped, the sample coated with the red insulating epoxy produced 1 larger red flake, 20 smaller red particles and 10 white particles. The specimen heated with an electric iron only exhibited 5 smaller particles. The sample coated with the black Dapco 2030 resulted in no flakes after being tapped.
\end{abstract}




\section{Introduction*}

Rohacell is a light, rigid, high quality polymethacrylimide foam. The most impressive feature is its strength to weight characteristics when compared to styrene and polyurethane foams. Rohacell's high compression modulus makes it an ideal choice for use as a core in structures requiring high strength (2). In addition to the high compression modulus, Rohacell's low coefficient of thermal conductivity makes it a good choice for cryogenic applications, like the VLPC cryostat cassettes (1). Machining Rohacell requires using the same techniques as machining wood. Rohacell should not be hot-wired, since the formation of harmful vapors is possible. Rohacell is compatible with all types of adhesives and finishes excluding those containing alkaline (2).

For the DO upgrade, Rohacell is an insulator in the cassettes for the VLPC cryostat. The problem detected was that the Rohacell tended to flake. This presented a problem because the particles of Rohacell could affect data by interfering with the electrical equipment surrounding it. The goal was to find a way to reduce or prevent the Rohacell from flaking. Initially, there were thoughts that cleaning the Rohacell with distilled water or rubbing alcohol, or spraying the Rohacell with a protective coating such as Krylon could reduce the amount of particles. After some research on Rohacell and calling the supplier for suggestions, it was certain; cleaning the Rohacell would not be a probable solution. The supplier, Richmond Aircraft Products, recommended using a two-part epoxy to prevent flaking. This suggestion reinforced the information found on the Internet concerning Rohacell.

Information found on parylene, a generic name for a unique family of polymers, seemed like the perfect solution to the problem (3). Eric Bosze of Los Alamos National Laboratory and the University of California in Riverside found himself dealing with the flaking of Rohacell also. His conclusions on the subject are found in his report, "Rohacell foam as a silicon support structure material for the PHENIX multiplicity vertex detector." $\mathrm{He}$ determined that using a 0.5-mil coating of parylene enhanced the strength and abrasion resistance, and acted as an environmental barrier for the

\footnotetext{
"Numbers in parenthesis refer to references on the reference page.
} 
Rohacell (3). During the application of the parylene, the flakes were collected and counted on black paper. As the thickness of the parylene increased, the amount of flakes decreased. In Figure 1, the non-linear relationship between the number of flakes and the thickness of the parylene is evident (3). Figure 1 also shows that the number of flakes starts to level out at 0.5 -mil of parylene (3). In locating more information on parylene and its application process, it was discovered that parylene is traditionally applied by vacuum deposition. This turns out to be an expensive and impractical method for our purpose.

Avg. \# of Flakes vs. Thickness of Coating

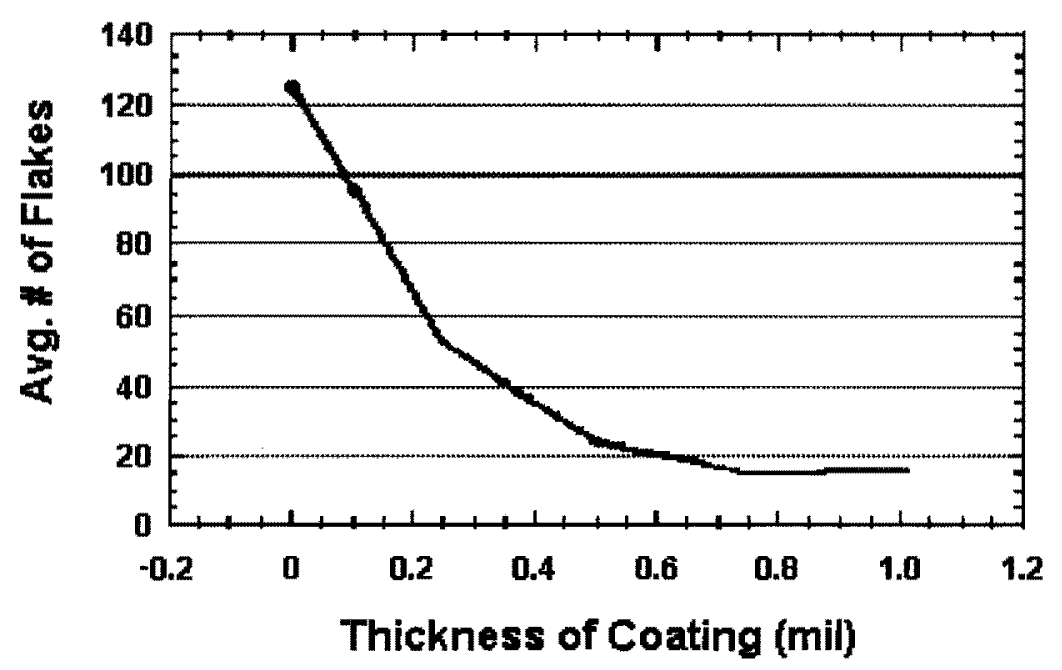

Results from Eric Bosze's Study on the Flaking of Rohacell

Figure 1

Further research resulted in the discovery of Dapco 2030, a cryogenic sealant and thermal insulation coating. Dapco 2030 is a two-component, flouroelastomer compound with excellent cryogenic properties. Traditional use of this compound is in areas requiring a coating for very low temperatures and chemical resistance (See Appendix A for further information) (6). 


\section{Procedure}

The Rohacell specimens were 3 inches long, 1.25 inches wide and 0.25 inches thick. The first four samples went untreated before the application of the testing agents. The testing agents for the first test included; one sprayed with Krylon ${ }^{\top M^{*}}$, one coated with ElectraMates ${ }^{\top M^{* *}}$ red Insulating Epoxy Coating, one sprayed with Vacseal's ${ }^{\top M}{ }^{\text {"* }}$ High Vacuum Leak Sealant, and one uncovered. Specimens five through eight, before applying the testing agents, were brushed in order to remove particles. The rinth sample went untouched before treating it with a heat gun. Samples ten and eleven were heated with an electric iron, one on medium and the other on high. Samples twelve and thirteen were coated with black Dapco 2030. The tapping of each specimen on dark paper 30 times, after being treated, tested for flakes. The Dapco 2030 samples were tapped on light paper as well as dark paper.

After this first test was complete, specimens five through eight and eleven through thirteen went through a cryogenic cycle. The cycle included submerging the specimens in liquid nitrogen then warming them to room temperature five times. The specimens withstood the tapping on the paper again to see how many particles came off. The same specimens endured the cycle and tapping one final time.

\footnotetext{
"Krylon is a trademark of and manufactured by Sherwin-Williams.

... ElectraMates is a trademark of and manufactured by Aervoe-Pacific Company, Inc.

... Vacseal is a trademark of and manufactured by Huntington Mechanical Laboratories, Inc
} 


\section{Test Summary of Rohacell Dust Project}

\begin{tabular}{|c|c|c|c|c|}
\hline Sample & Sealing Method & Particles before Cryo-cycle & Particles after first Cryo-cycle ${ }^{*}$ & Particles after second Cryo-cycle* \\
\hline 1 & Untreated & many particles & NA & NA \\
\hline 2 & Sprayed with Krylon & significantly less than sample 1 & NA & NA \\
\hline 3 & Vacuum Sealant & a little less than sample 2 & NA & NA \\
\hline 4 & Red Insulating Epoxy & $\begin{array}{l}\text { fewer particles than } 3 \text {, but include red and } \\
\text { white particles }\end{array}$ & NA & NA \\
\hline 5 & Untreated but brushed & many particles & $\begin{array}{l}4 \text { larger particles and a large } \\
\text { amount of smaller particles }\end{array}$ & $\begin{array}{c}8 \text { larger particles, } 20 \text { smaller } \\
\text { particles }\end{array}$ \\
\hline 6 & Brushed then Krylon & $\begin{array}{c}3 \text { larger white particles and } 15 \text { smaller white } \\
\text { particles }\end{array}$ & $\begin{array}{l}3 \text { larger particles and more smaller } \\
\text { particles than before Cryo-cycle }\end{array}$ & $\begin{array}{c}5 \text { larger particles, } 25 \text { smaller } \\
\text { particles }\end{array}$ \\
\hline 7 & $\begin{array}{l}\text { Brushed then Vacuum } \\
\text { Sealant }\end{array}$ & 10 white particles & $\begin{array}{c}2 \text { larger particles, } 10 \text { smaller } \\
\text { particles }\end{array}$ & 5 smaller particles \\
\hline 8 & $\begin{array}{l}\text { Brushed then Insulating } \\
\text { Epoxy }\end{array}$ & $\begin{array}{c}4 \text { large red particles, } 1 \text { white particle and } 10 \\
\text { small red particles }\end{array}$ & $\begin{array}{c}5 \text { larger red particles, } 15 \text { smaller red } \\
\text { particles, and } 3 \text { white particles }\end{array}$ & $\begin{array}{l}1 \text { larger red particle, } 20 \text { smaller red } \\
\text { particles, } 10 \text { white particles }\end{array}$ \\
\hline 9 & Heated with Heat Gun & 5 white particles & NA & NA \\
\hline 10 & $\begin{array}{l}\text { Heated with Iron on } \\
\text { Medium Heat }\end{array}$ & More particles than Krylon & NA & NA \\
\hline 11 & $\begin{array}{l}\text { Heated with Iron on } \\
\text { High Heat }\end{array}$ & several small particles & $\begin{array}{c}7 \text { larger particles, } 20 \text { smaller } \\
\text { particles }\end{array}$ & 5 smaller particles \\
\hline 12 & DAPCO 2030 & 4 black particles, no white particles & No white or black particles & No white or black particles \\
\hline 13 & DAPCO 2030 & No white or black particles & No white or black particles & No white or black particles \\
\hline
\end{tabular}

* After sealing, samples were submerged in liquid Nitrogen and warmed 5 times

Note: All samples were tapped 30 times on dark paper except the DAPCO 2030 which was tapped on dark and light paper

Table 1 


\section{Results}

The third column of Table 1 shows the first phase of the testing that left the samples untreated before coating and undergoing the tapping test. The first sample deposited a thin layer of particles on the paper. Sample two, sprayed with Krylon, had a significantly less amount of particles than sample one had. The third sample, protected by the vacuum sealant, had a substantially lesser amount of particles than sample one had and slightly smaller amount of particles than sample two had. Sample four, treated with the insulating epoxy, had both white and red particles after being tapped. Although the amount of particles from the fourth sample was considerably lower than the amounts from other tests, this presented the problem of epoxy flaking off the sample.

The same agents participated in the second set of samples, but the brushing of the samples to remove particles prepared them before the application of the coatings. Sample five still produced a numerous amount of particles on the paper, although there were fewer particles with the brushing than without brushing. The sixth sample, covered with Krylon, had 3 larger and 15 smaller white particles, a significantly fewer number of particles than sample five. The specimen coated with the vacuum sealant, sample seven, had approximately 10 white particles. This amount of flakes was remarkably smaller than what sample five and slightly less than the amount of particles from sample six. The eighth sample, protected with the insulating epoxy, had roughly 4 larger red particles, 10 smaller red particles, and 1 white particle on the dark paper. The amount of particles from sample eight is no worse than the amount off other specimens, but the epoxy flaking off was still a problem.

Another possible method was the use of heat to fuse the outer layer of particles together. Although there were several choices on how to do this, the decision was that a heat gun and an iron should treat the samples. Thoughts of putting the specimens into an oven were discarded quickly because the internal structure of the Rohacell could be compromised. It is possible that the thermal conductivity would change if the internal structure developed any damage. Sample nine was treated with a heat gun. Mixed 
results came from this heat-treated sample. On the one hand, only four flakes detached from this sample, but on the other hand, the specimen deformed during heated. An electric iron heated two other specimens, one on the medium setting and the other on the highest setting. Sample ten, ironed on medium, left a thin coat of particles on the dark paper. The second specimen, sample eleven, had much better results. There were only several small particles from this second iron treated sample. Neither samples ten nor eleven deformed in any way.

Samples twelve and thirteen were coated with the black Dapco 2030. After sample twelve was tapped on dark and light paper, there were 4 black particles and no white particles. Sample thirteen, when tapped, had no black or white particles on the paper.

The fourth column of Table 1 shows which samples were chosen to undergo the cryogenic cycle and the results of phase two of the testing. Sample five, after tapping, had 4 larger particles and a large amount of smaller particles. Following the cryogenic cycle and tapping, sample six left 3 larger flakes and 10 smaller particles. After testing sample seven there were 2 larger particles and 10 smaller particles. Sample eight left 5 larger red particles, 15 smaller red particles and 3 white particles after the tapping. The eleventh sample, after the testing, left 7 larger particles and 20 smaller particles. Both sample twelve and sample thirteen did not leave any particles behind.

The fifth colurnn of Table 1 shows the results of phase three of the testing. These samples underwent a second cryogenic cycle and then tapped again. Sample five had 8 larger flakes and 20 smaller particles after tapping. Although the amount of larger particles was greater than before the second cryogenic cycle, there was an evident reduction in the number of smaller particles. The sixth sample produced 5 larger particles and 25 smaller particles. Only 5 smaller particles detached from sample seven, a definite advantage over samples five and six. Sample eight had 1 large red particle, 20 smaller red particles and 10 white particles. Like sample seven, only five smaller particles detached from sample eleven. Again, both sample twelve and sample thirteen did not have particles detach while testing. 


\section{Conclusion}

In order to prevent the Rohacell from flaking, the best solution is the Dapco 2030. Dapco 2030 was the only coating not to have any flakes after the second cryogenic cycle. In using the Dapco 2030 as the sealant for the Rohacell, there would be no need to worry about particles interfering with the electrical equipment and affecting the data taken from the VLPC. Dapco 2030 would also be a good choice because it is black and would absorb light. Any light leaking in could interfere with the data.

Either ironing or spraying with the vacuum sealant after brushing would be the second best solution for flaking. Both of these methods only had 5 particles after the second cryogenic cycle. Of these two methods, ironing the Rohacell would be the most economical.

The problem with the Krylon and the red insulating epoxy is that they did not do very well after the cryogenic cycles. Before the cryogenic cycles these two coating had approximately the same number of particles coming off the samples. Just brushing the Rohacell is not a solution for preventing the flaking. Even after the sample was brushed, there was still a large amount of particles after the tapping. 


\section{References}

1.) www.tminet.com/cst/rohacell.htm

2.) www.deltronix.com/public/acp/acp-roha.htm

3.) http://p2hp2.lanl.gov/people/ebosze/papers/mvd/node3.html

4.) http://rsgi01.rhic.bnl.gov/phenixWWW/publish/hubert/mvd talk nov96/rohacell.html

5.) http://p2hp2.lanl.gov/people/ebosze/papers/mvd/nodeg.html

6.) http://www.d-aircraft.com/dapc2030.htm 


\section{APPENDIX A}

Information on Dapco 2030 


\section{DAPCO 2030 \\ CRYOGENIC SEALANT \\ THERMAL INSULATION COATING*}

\section{GENERAL DESCRIPTION}

Dapco 2030 is a two-component, flouroelastomer compound with the following features:

- Excellent LOX compatibility

- Good cryogenic properties

- Non-burning

- Resistance to aircraft fuels and fluids

- Resistance to hydrazine

\section{APPLICATIONS}

Dapco 2030 is commonly used in areas that require a coating for very low and/or very high temperature and chemical resistance.

Dapco 2030 renders polyurethane and epoxy foams self-extinguishing.

Dapco 2030 can also be used to seal the backside of porous tools for vacuum retention.

TYPICAL PROPERTIES

Color

Solids, \%

Appearance

Viscosity (cps @72F)

Density, Ibs./gal
PART A
White*
$30-35$
Liquid
2,000

8.8

PART B
Yellow
1
Liquid
5

6.8
MIXED

White*

30-35

Liquid

2,000

8.2

*Different colors are available. Varies with color.

\footnotetext{
- All the information from this section is taken from the website: www.d-aircraft.com/dapc2030.htm
} 


\section{PROCESSING}

Mix Ratio: The recommended mix ratio for Dapco 2030 is:

$\begin{array}{cc} & \text { Weight } \\ \text { Part A } & 100 \\ \text { Part B } & 5\end{array}$

Combine Part $A$ and Part $B$ and mix well. Allow mixture to stand one to two hours before use.

Dapco 2030 can be applied by brush or dip method as supplied. Apply thin, uniform coats. Allow 15 to 30 minutes between coats.

For spray application, thin Dapco 2030 with MEK or MIBK to proper consistency, depending on equipment used.

Working Life: The pot life of Dapco 2030 after mixing is at least eight hours.

Curing: Dapco 2030 achieves optimum cured properties when cured at room temperature (7OF) for three to five days.

Optional Curing: Dapco 2030 may also be cured by using heat. The following step cure is recommended to avoid solvent entrapment and blistering:

- 4 hours at room temp (70F) (after solvent flash off)

- 8 hours at $120 \mathrm{~F}$

- 2 hours at $150 \mathrm{~F}$

- 1 hour at $200 \mathrm{~F}$

\section{SURFACE PREPARATION}

The surface(s) must be free from contaminates, i.e., dirt, oil, grease, etc. Clean the surface by wiping with a suitable solvent/cleaning agent and dry thoroughly.

\section{TYPICAL CURED PROPERTIES}

Tensile Strength, $\quad 1000$ psi

Elongation, $\quad 300 \%$

Adhesion, $\quad>4$ by tape test ASTM D-3359-87 
STORAGE AND HANDLING

Store in a cool dry place.

Keep containers tightly sealed.

\section{SAFETY}

Contains flammable solvents. Keep away from open flame and excessive heat. Use with adequate ventilation.

Exercise good housekeeping practices. Material Safety Data Sheets available upon request.

\section{Cost}

Quart

$\$ 124.80$

Gallon $\quad \$ 390.40$ 